\title{
Superior Artery Mesenteric Syndrome
}

Dr. Esteban Salas Salas . ${ }^{1}$ Dr. José Pablo Jiménez Trigueros ${ }^{2}$

1 Médico cirujano especialista en cirugía general. Caja costarricense de seguro social, Puntarenas Costa Rica. 2 Médico cirujano especialista en cirugía general. Caja costarricense de seguro social Limón, Costa Rica

Contacto: salasesteban@gmail.com

\section{Resumen}

El síndrome de arteria mesentérica superior o también llamado síndrome de Wilkie, consiste en un diagnóstico de exclusión, ya que es una condición clínica que causa un cuadro de obstrucción intestinal alto de forma crónica usualmente debido a un acortamiento del ángulo aorto mesentérico y la distancia aortomesentérica. Su diagnóstico es usualmente difícil de realizar para el médico tratante ya que es una patología poco frecuente. Su clínica se basa en vómitos postprandiales, llenura postprandial, pérdida de peso y suele acompañarse de condiciones adicionales que causan un franco adelgazamiento del paciente. En cuanto a la valoración clínica, el manejo multidisciplinario es fundamental e involucra múltiples especialidades médicas y quirúrgicas. El manejo inicial es médico, asociado a un soporte nutricional importante, el cual se basa en lograr un claro aumento de peso y masa muscular; sin embargo, muchas veces es fallido y el paciente requiere de cirugía. La valoración psiquiátrica es también muy importante debido a que muchas veces se asocia con patologías como trastornos alimentarios. Una vez que el grupo interdisciplinario ha determinado que el manejo médico es insuficiente, debe valorarse la cirugía. En este caso, actualmente la técnica ideal con menos morbilidad asociada y mayor resolución de sintomatología consiste en la duodeno yeyunostomía laparoscópica. La cirugía robótica también ha surgido como otra técnica quirúrgica para realizar este procedimiento; no obstante, debido a su alta complejidad y preparación del personal aún se encuentran realizando estudios aleatorizados controlados para comparar ambas técnicas.

Palabras clave:

Síndrome de arteria mesentérica superior; obstrucción intestinal; duodenoyeyunostomía; dolor abdominal

\section{Abstract}

The superior mesenteric artery syndrome or also called Wilkie syndrome is a diagnosis of

Recibido: 21/oct/2020

Aceptado: 06/nov/2020

Publicado: 15/dic/2020 exclusion, this is because it consists of a condition that causes a clinical condition of chronic high intestinal obstruction due to a shortening of the aortomesenteric angle and the aortomesenteric distance. The diagnosis is usually very difficult to achieve to the physician because it is a condition pretty uncommon. The clinic of this condition is based in postprandial vomits, loss of appetite and it usually accompanies other conditions that cause a severe loss of weight of the patient. In the clinical consult, the multidisciplinary management is fundamental, and it requires multiple medical and surgical specialties.. The initial management is basically medical and with nutritional support and is based on a rise in the weight of the patient, principally with muscular mass, although many times it fails and the patient requires surgery. One very important point consists in the psychiatric consult, because in many cases this condition is associated with mental sickness and alimentary disorders. Once the multidisciplinary treatment group has decided that the medical treatment is insufficient, Keywords:

Superior mesenteric artery syndrome; intestinal obstruction; duodenojejunostomy; abdominal pain 
the patient may require a surgical consult. When this happens the ideal technique with the less morbidity and major resolution of symptoms consist in the laparoscopic duodenojejunostomy. The robotical technique has also emerged as an other option for this type of procedure, although, the medical preparation and the complexity is nowadays in debate and more randomized studies are necessary to determine the efficacy of this technique.

\section{INTRODUCCIÓN}

El síndrome de arteria mesentérica superior (SAMS) o también llamado síndrome de Wilkie, es una patología gastrointestinal poco frecuente que ocasiona un cuadro de obstrucción intestinal de forma crónica (1). Fue descrito por Rokitanski en 1861 y Wilkie publica la primera serie de 75 pacientes en 1927 (2). Su mecanismo fisiopatológico se basa en una compresión mecánica extrínseca de la tercera porción de duodeno, debido a que existe un curso anómalo de la arteria mesentérica superior de acuerdo con su origen desde la aorta abdominal. Dicha anomalía causa que se reduzca el ángulo normal de la misma, por lo que se provoca indirectamente una obstrucción entre la tercera y cuarta porción del duodeno, asociado con una pérdida de la grasa mesentérica que potencia aún más dicha condición (1). Su incidencia en términos generales de acuerdo con los estudios actuales se encuentra entre el 0.1 al $0.3 \%$, ocurre más frecuentemente en mujeres que en hombres, con índice aproximado de 3:2, y a menudo afecta a jóvenes de 10 a 39 años, aunque puede existir a cualquier edad, sin embargo su incidencia real se desconoce $(1,2)$.

SAMS se presenta típicamente en mujeres jóvenes entre los 10 y 40 años en las cuales deben excluirse otros trastornos tales como: síndrome de vómitos cíclico, gastroparesia, dispepsia funcional o gastritis debido a Helicobacter pylori. Además, por otro lado, SAMS también se ha ligado a desórdenes psiquiátricos con síntomas digestivos asociados como anorexia o bulimia (3).

En cuanto a la etiología, esta puede ser congénita o relacionada con factores adquiridos, tales como pérdidas repentinas de peso (cirugía bariátrica), elongación de la columna vertebral en escoliosis o posterior a alguna cirugía abdominal de mayor complejidad como por ejemplo proctocolectomía con reservorio ileal (1). Asimismo, el trauma se ha ligado a esta condición y debe ser tomao en cuenta dentro de los factores adquiridos (1).

El objetivo de la presente revisión consiste en lograr determinar el tratamiento efectivo y adecuado para el síndrome de arteria mesentérica superior.

\section{MÉTODO}

Durante la realización de esta revisión bibliográfica, se utilizó el buscador médico en PUBMED y DYNAMED, para encontrar bibliografía que date con fechas no mayores a los seis años, para lo anterior se colocaron los términos "síndrome de arteria mesentérica superior" y "duodeno yeyunostomía laparoscópica", tanto en los idiomas español como inglés. De esta manera se utilizaron como criterios de inclusión en dicha búsqueda revisiones científicas que han logrado establecer el mejor método de tratamiento de esta patología. Por otro lado, dicha búsqueda se enfocó en revisar el manejo quirúrgico actual de SAMS dirigido hacia la cirugía de tipo duodeno yeyunostomía laparoscópica.

\section{PRESENTACIÓN CLÍNICA}

En cuanto al diagnóstico de SAMS, es importante recordar que usualmente suele ser un diagnóstico de exclusión, ya que no es una causa común de obstrucción intestinal y más específicamente duodenal crónica. Para lograr su adecuado diagnóstico se toman en cuenta aquellos pacientes que presentan síntomas de obstrucción intestinal crónica de al menos cuatro semanas, que suelen ser: vómitos postprandiales, sensación de llenura temprana, pérdida de peso o incapacidad para aumentar de peso $(1,2)$. Muchas veces los pacientes refieren dolor abdominal recurrente que se empeora en posición supina y se alivia cuando se elevan las rodillas sobre el pecho (maniobra en la cual se aumenta el ángulo aorto mesentérico y por ende se alivian parcialmente los síntomas de obstrucción) (3). En cuanto a los hallazgos al examen físico, estos suelen ser sumamente inespecíficos como adelgazamiento y pérdida de masa muscular por la desnutrición asociada de fondo, dolor abdominal en epigastrio inespecífico o datos de deshidratación crónica por los vómitos postprandiales (2). Por otro lado, es 
importante recalcar que debido a su difícil diagnóstico, usualmente este se logra realizar de manera tardía, momento en el cual el paciente se encuentra sumamente adelgazado y este hecho afecta asimismo la adecuada resolución de los síntomas por medio de la cirugía (1).

\section{DIAGNÓSTICO}

El diagnóstico es usualmente complejo de realizar para el clínico y es de forma multidisciplinaria debido a que involucra tanto lo radiológico como los aspectos clínicos según la evolución del paciente. Dicha sintomatología debe acompañarse de una evaluación radiológica adecuada representada como: dilatación duodenal con dilatación gástrica y estasis del contenido alimentario. En muchas ocasiones, es útil la realización de estudios contrastados dinámicos para visualizar el tránsito gastrointestinal y así lograr demostrar un tránsito lento o un paso tórpido entre la tercera y cuarta porción duodenal. La medición adecuada del ángulo y distancia aortomesentérica es sumamente importante, con medidas menores o iguales a $25^{\circ}$ y $8 \mathrm{~mm}$ respectivamente. Esta distancia debe ser determinada por un profesional médico en radiología por medio de una Tomografía Axial Computarizada con medio intravenoso $(4,5)$.

En cuanto a la exclusión de otras patologías médicas es importante considerar la realización de estudios complementarios como: endoscopía digestiva alta, ultrasonografías abdominales y manometría esofágica (2). Se recalca que el diagnóstico de este tipo de patología debe ser multidisciplinario, al incluir especialidades médicas y quirúrgicas, por ejemplo: cirugía general, gastroenterología, radiología, medicina interna y psiquiatría (1). Ante dichos hallazgos, lograr un adecuado diagnóstico de SAMS suele ser algo muy complejo y deben tenerse en cuenta muchos factores clínicos que se integren de forma adecuada para lograr resolver de la mejor forma la patología que presente el paciente $(1,2)$.

\section{MANEJO CONSERVADOR}

En un porcentaje considerable de los casos, los pacientes que se logran diagnosticar con este tipo de patología no llegan a requerir cirugía (5). Esto se debe a que el SAMS se encuentra relacionado con cuadros de pérdida de peso repentino y súbito, en los cuales existe una franca pérdida de la grasa que se encuentra en el ángulo aortomesentérico y esta al desaparecer, ocasiona un acortamiento de dicho ángulo con el pinzamiento consecuente de la arteria mesentérica superior sobre la aorta, con lo cual se origina la compresión de la tercera porción duodenal y los síntomas descritos para cada paciente $(6,7)$. Ante dichos hallazgos descritos, existe un porcentaje considerable de pacientes en los cuales solamente con aumentar el requerimiento proteico y calórico, pueden llegar a resolver su sintomatología y su cuadro clínico (8). El manejo conservador del SAMS tiene dos aristas principales, las cuales consisten en el soporte clínico y nutricional, y por otro lado el enfoque desde el punto de vista psicológico o muchas veces psiquiátrico. Una vez que se establece el diagnóstico de SAMS, todo paciente (independientemente de si llegue a requerir manejo quirúrgico o no) debe ser valorado integralmente desde el punto de vista nutricional por un equipo multidisciplinario con el fin de determinar la mejor ruta de alimentación para el paciente $(6,7)$. Cuando el paciente es diagnosticado de manera aguda, es importante ingresarlo a un servicio de hospitalización médica para iniciar una serie de medidas tales como: corrección de la deshidratación y trastornos electrolíticos asociados, así como asegurar una forma de alimentación adecuada para el paciente $(8,9)$.

Muchas veces este tipo de pacientes amerita el uso de nutrición enteral por medio de sondas de alimentación nasoenterales, debido a la intolerancia a la vía oral. En otros casos, no es necesaria la utilización de este tipo de dispositivos ya que aún el paciente es capaz de deglutir y comer cierta cantidad de alimentos, ante dichos casos lo que se procede a realizar es una valoración integral nutricional para determinar el uso de nutrición parenteral y así lograr un aumento de peso adecuado para el paciente (6). La vía de alimentación preferible consiste en la enteral, ya que permite una mejor respuesta ante los efectos tróficos en la mucosa gastrointestinal $(1,2)$.

En resumen, los pilares del manejo conservador son los siguientes $(8,9,12)$ :

- Valoración por psiquiatría y nutrición.

- $\quad$ Alimentación adecuada y oportuna del paciente (uso de sondas naso enterales en caso necesario).

. $\quad$ Asegurarse por parte del médico tratante la integridad del tracto digestivo.

- Corrección de la deshidratación y los trastornos electrolíticos (hipocalemia, hiponatremia).

- Hospitalización en caso necesario para un abordaje integral y multidisciplinario que logre cumplir todas las medidas para un aumento de peso y de masa muscular. 


\section{MANEJO QUIRÚRGICO}

En ocasiones, una vez iniciada la estabilización clínica y nutricional del paciente, este sigue presentado la sintomatología que lo hizo consultar en la primera ocasión, ante esta situación y la incapacidad para el adecuado aumento de peso se debe considerar la opción quirúrgica $(8,9)$. Todo paciente que llegue a requerir manejo quirúrgico, inicialmente debe someterse a una valoración clínica y nutricional como la descrita en el manejo conservador para lograr mejorar su condición nutricional y obtener mejores resultados posteriormente $(9,10)$. Es importante mencionar que el equipo médico y nutricional a cargo del paciente debe tomar la decisión en conjunto acerca del fallo en el manejo conservador, ya que muchas veces esta decisión se toma sin contemplar las complicaciones de un procedimiento quirúrgico (8). El porcentaje de pacientes que requiere cirugía se encuentra en un rango entre $10 \%$ a $15 \%$, ya que la cirugía tiene sus morbilidades asociadas y además el tratamiento conservador también ha demostrado tener efectos beneficiosos en cuanto a la resolución de la cirugía $(10,11)$.

En cuanto al tipo de cirugía que se le proponga al paciente, la técnica mínimamente invasiva puede presentarse como una adecuada alternativa, especialmente en pacientes que se encuentren debilitados por alguna otra condición clínica $(11,12)$. Las tres principales opciones quirúrgicas descritas en la literatura actualmente que se realizan con mayor frecuencia y con mejores resultados para el alivio de los síntomas consisten en (9):

1. Procedimiento de Strong.

2. Gastroyeyunostomía abierta versus laparoscópica.

3. Duodenoyeyunostomía ya sea de forma abierta o con técnica mínimamente invasiva como la laparoscopía.

La operación propuesta por Strong en el año 1958 fue la primera técnica en ser utilizada para este tipo de padecimiento y consiste en la sección del ligamento de Treitz y la posterior movilización del duodeno para evitar su pinzamiento entre la arteria mesentérica superior y la aorta; sin embargo debido a la morbilidad asociada prácticamente no se utiliza, además presenta una tasa de fracaso sumamente alta, de hasta un $25 \%(1,12)$. Por otro lado, la gastroyeyunostomía ha demostrado una recurrencia considerable de la sintomatología, debido a su relación con el importante reflujo alcalino y un síndrome de obstrucción de asa cerrada (12). De esta manera, dentro de todas las técnicas quirúrgicas disponibles, la que ha demostrado mayor efectividad en la resolución de los síntomas consiste en la duodenoyeyunostomía, con una tasa de éxito superior al $90 \%(10,11,12)$.

En 1998, Bernas y Richardson describieron la duodenoyeyunostomía de forma laparoscópica como una alternativa eficaz y segura con menor morbilidad en comparación con la técnica abierta (2). La anastomosis en estos casos es usualmente latero-lateral, por debajo del colon transverso, y a la derecha del mesocolon. Un punto importante es que no existe un consenso claro en la literatura acerca de la sección o no del duodeno en su cuarta porción, de manera que se han demostrado anastomosis satisfactorias sin realizar este paso $(1,2,13)$. De esta manera, en las últimas revisiones con mayores efectos positivos para el paciente, Munene describió la realización de duodenoyeyunostomía laparoscópica en 13 pacientes con y sin sección de la cuarta porción duodenal $(13,14)$. Es así como demostró una adecuada identificación del campo quirúrgico y su efectividad en cuanto a la laparoscopía (13). Recientemente, Guilanoti, et al., reportaron el uso de la cirugía robótica para lograr la anastomosis de una forma totalmente manual, lo cual puede ser muy desafiante si se intenta realizar de forma laparoscópica $(9,10)$.

En cuanto a la descripción de la técnica laparoscópica, esta deber ser realizada por un cirujano con experiencia en cirugía mínimamente invasiva, en la cual el paciente se somete a anestesia general, en posición de Trendelemburg reversa $\left(20^{\circ}\right.$ a $\left.30^{\circ}\right)$, el cirujano accede al neumoperitoneo con su técnica de preferencia. Se logra acceso a la cavidad abdominal y se colocan dos trocares adicionales de $5 \mathrm{~mm}$, uno debajo del inicial y otro en flanco izquierdo y posteriormente otro tercero en fosa ilíaca derecha. Una vez que se identifica el omento mayor y el mesocolon, debe realizarse tracción craneal para lograr exponer el duodeno hacia el ligamento de Treitz y el yeyuno. Posteriormente con un cauterio en forma de gancho debe separarse el peritoneo visceral que cubre la segunda y tercera porción del duodeno y se separa cuidadosamente el duodeno del retroperitoneo para lograr facilitar la anastomosis. Se identifica un asa de yeyuno 20 a $30 \mathrm{~cm}$ distal al ligamento de Treitz, se realiza una enterotomía en el duodeno y en el yeyuno para realizar una anastomosis lateral mecánica con el cierre de la misma con sutura barbada. No es necesario el uso de sondas nasogástricas o drenajes intraabdominales. La tolerancia a líquidos se puede permitir al primer día postoperatorio $(1,2,15)$. 


\section{CONCLUSIÓN}

EI SAMS es un diagnóstico de exclusión que debe ser tomado en cuenta a la hora de valorar un paciente con un cuadro de obstrucción intestinal alta y que además ha perdido peso sustancialmente, su clínica es principalmente vómitos postprandiales y dolor abdominal recurrente. Es importante recalcar que su diagnóstico es usualmente de exclusión, por lo tanto deben descartarse múltiples causas de obstrucción intestinal que tienen mayor incidencia previo a realizar este diagnóstico. Su fisiopatología se basa en la disminución del ángulo y la distancia aortomesentérica; asociado con condiciones que favorecen la pérdida de peso repentina o situaciones en las cuales el paciente ha experimentado una muy pobre capacidad para aumentar de peso; para lo cual el diagnóstico y tratamiento debe ser multidisciplinario. El tratamiento de esta patología usualmente no requiere cirugía y su abordaje inicial implica un adecuado soporte nutricional para lograr que el paciente aumente su índice de masa corporal y el ángulo aorto mesentérico se amplié y así disminuir el cuadro obstructivo. Cuando el manejo conservador falla, el paciente debe ser llevado a cirugía para una duodeno yeyunostomía laparoscópica como mejor opción, ya que este procedimiento ha logrado demostrar una tasa de éxito amplia con el menor índice de complicaciones pos operatorias asociadas. Como recomendaciones a futuro, es importante anotar que la duodenoyeyunostomía laparoscópica sigue en estudio para poder ser establecida como el procedimiento quirúrgico ideal en este tipo de pacientes, sin embargo debido a que es una patología poco frecuente, es importante realizar más estudios aleatorizados comparando las diferentes técnicas quirúrgicas. Por otro lado, la cirugía robótica continúa siendo un pilar a tomar en cuenta y amerita mayor estudio para determinar si verdaderamente es más eficiente que la laparoscopía en este tipo de patología. Además, es importante realizar más investigación para lograr establecer el mejor tratamiento en los pacientes, ya sea la diferencia entre el quirúrgico y conservador y claras indicaciones hacia uno a otro.

\section{REFERENCIAS BIBLIOGRÁFICAS}

1. Cienfuegos, J., Hurtado-Pardo, L., Valenti, V., Landecho, M., Vivas, I., Estévez, M., Diez-Caballero, A., Hernández-Lizoáin, J. and Rotellar, F., 2020. Minimally Invasive Surgical Approach for the Treatment of Superior Mesenteric Artery Syndrome: Long-Term Outcomes. World Journal of Surgery. Disponible en: https://link.springer.com/article/10.1007/s00268-02005413-5

2. Barchi L, 4. Martínez H, Martínez S, Sánchez-Ussa S, Pedraza M, Cabrera L. Manejo laparoscópico para el síndrome de Wilkie. Cirugía y Cirujanos. 2019;87(91). Disponible en: https://www.medigraphic.com/cgi-bin/new/resumen.cgi?IDARTICULO $=89108$

3. Barchi L, Alves A, Jacob C, Caldas Bresciani C, Yagi O, Nogueira $T$ et al. Favorable minimal invasive surgery in the treatment of superior mesenteric artery syndrome: Case report. International Journal of Surgery Case Reports. 2016;29:223226. Disponible en: https://pubmed.ncbi.n/m.nih.gov/27914348

4. Alves A, Jacob C, Caldas Bresciani C, Yagi O, Nogueira T et al. Favorable minimal invasive surgery in the treatment of superior mesenteric artery syndrome: Case report. International Journal of Surgery Case Reports. 2016;29:223-226. Disponible en: https://www.sciencedirect.com/science/article/pii/S2210261216303571

5. Salem A, Al Ozaibi L, Nassif S, Osman R, Al Abed N, Badri F. Superior mesenteric artery syndrome: A diagnosis to be kept in mind (Case report and literature review). International Journal of Surgery Case Reports. 2017;34:84-86. Disponible en: https://pubmed.ncbi.nlm.nih.gov/28376419/

6. Barkhatov L, Tyukina N, Fretland $\AA$, Røsok B, Kazaryan A, Riis $R$ et al. Superior mesenteric artery syndrome: quality of life after laparoscopic duodenojejunostomy. Clinical Case Reports. 2017;6(2):323-329. Disponible en: https://pubmed.ncbi. nlm.nih.gov/29445471/

7. Ganss A, Rampado S, Savarino E, Bardini R. Superior Mesenteric Artery Syndrome: a Prospective Study in a Sin- 


\section{CIENCIA\&SALUD}

gle Institution. Journal of Gastrointestinal Surgery. 2018;23(5):997-1005. Disponible en: https://link.springer.com/article/10.1007\%2Fs11605-018-3984-6

8. Chang J, Boules M, Rodriguez J, Walsh M, Rosenthal R, Kroh M. Laparoscopic duodenojejunostomy for superior mesenteric artery syndrome: intermediate follow-up results and a review of the literature. Surgical Endoscopy. 2016;31(3):11801185. Disponible en: $h$ ttps://link.springer.com/article/10.1007\%2Fs00464-016-5088-2

9. Yoneda A, Kanetaka K, Yamaguchi S, Koga Y, Isagawa Y, Maruya Y et al. Complete laparoscopic duodenojejunostomy for superior mesenteric artery syndrome: Linear stapled closure of the common enterotomy. Asian Journal of Endoscopic Surgery. 2019. Disponible en: https://pubmed.ncbi.n/m.nih.gov/20349665/

10. Bardini R. Superior Mesenteric Artery Syndrome: Author's Point of View. Journal of Gastrointestinal Surgery. 2019;24(2):446-447. Disponible en: https://link.springer.com/article/10.1007\%2Fs11605-019-04453-0

11. Kirby G, Faulconer E, Robinson S, Perry A, Downing R. Superior mesenteric artery syndrome: a single centre experience of laparoscopic duodenojejunostomy as the operation of choice. The Annals of The Royal College of Surgeons of England. 2017;99(6):472-475. Disponible en: https://publishing.rcseng.ac.uk/doi/full/10.1308/rcsann.2017.0063?url_ ver=Z39.88-2003\&rfr_id=ori:rid:crossref.org\&rfr_dat=cr_pub\%20\%200pubmed

12. Yan $\mathrm{C}, \mathrm{Hu} Z$, Wu J, Zhang $C$, Yan L, Wang Z. Laparoscopic Toupet fundoplication with duodenojejunostomy for the management of superior mesenteric artery syndrome with reflux symptoms. Medicine. 2017;96(3):e5771. Disponible en: https://www.ncbi.nlm.nih.gov/pmc/articles/PMC5279079/

13. Agha R, Fowler A, Saeta A, Barai I, Rajmohan S, Orgill D et al. The SCARE Statement: Consensus-based surgical case report guidelines. International Journal of Surgery. 2016;34:180-186. Disponible en: https://www.sciencedirect.com/science/ article/pii/S174391911630303X?via\%3Dihub

14. Kaur A. Superior Mesentric Artery Syndrome in a Patient with Subacute Intestinal Obstruction: A Case Repor t. Journal of Clinical and Diagnostic Research. 2016. Disponible en: https://www.researchgate.net/publication/304708957_Superior_Mesentric_Artery_Syndrome_in_a_Patient_with_Subacute_Intestinal_Obstruction_A_Case_Repor_t

15. Lacy B, Parkman H, Camilleri M. Chronic nausea and vomiting: evaluation and treatment. American Journal of Gastroenterology. 2018;113(5):647-659. Disponible en: https://insights.ovid.com/pubmed?pmid=29545633 